Cahiers $d u$ MONDE RUSSE

\section{Cahiers du monde russe}

Russie - Empire russe - Union soviétique et États indépendants

$44 / 2-3 \mid 2003$

Les pratiques administratives en Union soviétique, 1920-1960

\title{
Diplomatie et diplomates soviétiques à l'ère du dégel 1953-1964
}

Marie-Pierre Rey

\section{OpenEdition}

Journals

Édition électronique

URL : https://journals.openedition.org/monderusse/8611

DOI : 10.4000/monderusse. 8611

ISSN : $1777-5388$

Éditeur

Éditions de l'EHESS

Édition imprimée

Date de publication : 1 avril 2003

Pagination : 309-322

ISBN : 2-7132-1813-6

ISSN : $1252-6576$

Référence électronique

Marie-Pierre Rey, "Diplomatie et diplomates soviétiques à l'ère du dégel 1953-1964 », Cahiers du monde russe [En ligne], 44/2-3 | 2003, mis en ligne le 01 janvier 2007, consulté le 03 septembre 2022 URL : http://journals.openedition.org/monderusse/8611; DOI : https://doi.org/10.4000/monderusse. 8611 


\section{CAIR N}

chercher : repérer : avancer

Cet article est disponible en ligne à l'adresse :

http://www.cairn.info/article.php?ID REVUE=CMR\&ID NUMPUBLIE=CMR 442\&ID ARTICLE=CMR 4420309

Diplomatie et diplomates soviétiques à l'ère du dégel 1953-1964

par Marie-Pierre REY

| Editions de l'EHESS | Cahiers du monde russe

2003/2-3 Vol 44

ISSN 1252-6576 | ISBN 2713218136 | pages 309 à 322

Pour citer cet article :

- REY M.-P., Diplomatie et diplomates soviétiques à l'ère du dégel 1953-1964, Cahiers du monde russe 2003/2, Vol 44, p. 309-322.

Distribution électronique Cairn pour les Editions de l'EHESS.

(C) Editions de l'EHESS. Tous droits réservés pour tous pays.

La reproduction ou représentation de cet article, notamment par photocopie, n'est autorisée que dans les limites des conditions générales d'utilisation du site ou, le cas échéant, des conditions générales de la licence souscrite par votre établissement. Toute autre reproduction ou représentation, en tout ou partie, sous quelque forme et de quelque manière que ce soit, est interdite sauf accord préalable et écrit de l'éditeur, en dehors des cas prévus par la législation en vigueur en France. Il est précisé que son stockage dans une base de données est également interdit. 


\section{DIPLOMATIE ET DIPLOMATES SOVIÉTIQUES À L'ÈRE DU DÉGEL 1953-1964}

Administration patiemment construite au fil des années 20 et 30, d'abord sous la houlette de Georgij Čičerin, puis de Maksim Litvinov, le commissariat du peuple aux Affaires étrangères, le Narkomindel (Narodnyj Komissariat Inostrannyh Del), subit de plein fouet les purges de la seconde moitié des années 30 et c'est une structure profondément transformée qui peu à peu émerge du cataclysme. Centralisé et hiérarchisé, étroitement soumis aux instances dirigeantes, en l'occurrence Stalin et Molotov, le Narkomindel a désormais perdu la petite marge de manœuvre qu'il avait réussi à se ménager dans la première moitié des années $30^{1}$.

Au lendemain de la Seconde Guerre mondiale, la situation évolue quelque peu. En 1946, la substitution du ministère des Affaires étrangères, le MID (Ministerstvo Inostrannyh Del), au Narkomindel, participe au renouveau d'une instance diplomatique qui retrouve un peu de son identité perdue. Mais jusqu'en 1953, le nouveau ministère reste étroitement dépendant des volontés personnelles de Stalin. Formés à l'École supérieure de la diplomatie, les diplomates soviétiques apparaissent alors comme des exécutants dociles et disciplinés, dépourvus de toute initiative: l'empreinte des purges reste très vivace.

Les années post-staliniennes et plus encore les années Hruščev à partir de 1956 voient des changements majeurs se dessiner dans la politique extérieure soviétique : la guerre froide fait place à la coexistence pacifique qui, elle-même, s'incarne dans des mesures très concrètes. Il suffit de rappeler la signature, en 1955, du traité d'État redonnant à l'Autriche sa souveraineté puis, en 1963, la signature du traité de Moscou sur l'interdiction partielle des essais nucléaires. Parallèlement, des

1. Voir Teddy J. Uldricks, « The impact of the Great Purges on the People's Commissariat of Foreign Affairs », Slavic Review, 36, 2, June 1977, p. 187-204 et l'ouvrage de Sabine Dullin, Des hommes d'influences, les ambassadeurs de Staline en Europe, 1930-1939, Paris, Payot, 2001. 
thématiques nouvelles font leur apparition, témoignant là encore de l'effervescence intellectuelle et conceptuelle qui anime l'appareil dirigeant.

Dans ce contexte de changement, que devient l'héritage diplomatique de la période stalinienne ? évolue-t-il ? se modifie-t-il ? En un mot, observe-t-on dans la période khrouchtchevienne des changements de nature quant à l'organisation et au fonctionnement de la politique extérieure ? quant aux perceptions et aux pratiques diplomatiques ? Enfin, le milieu des diplomates évolue-t-il aussi ?

Pour tenter de répondre à ces diverses questions, cet article distinguera deux parties. Dans un premier temps, l'on cherchera à cerner le rôle joué par l'administration du MID dans la formulation et l'exécution de la politique extérieure soviétique, ce qui nous amènera à traiter des relations cruciales entretenues par le MID et le PCUS tout au long de la période 1953-1964. Puis l'on s'intéressera à la machine diplomatique et aux hommes qui la composent,en s'interrogeant tout particulièrement sur son degré d'efficacité.

\section{Le rôle du MID dans l'élaboration et la mise en œuvre de la politique extérieure soviétique}

De la mort de Stalin à juin 1956, date à laquelle il est contraint de céder la place à Šepilov, Molotov est encore aux commandes. Dans cette période troublée, il gère le MID dans une continuité certaine par rapport à la fin des années 30, tout en s'efforçant de rendre à l'appareil diplomatique une part de l'autonomie qui était la sienne avant les grandes purges.

\section{3-1955, une nouvelle donne pour le MID ?}

Ponctuées par des rivalités au sein de l'appareil dirigeant, les années 1953-1954 sont particulièrement crispées et la politique étrangère n'échappe pas à ces tensions. Plusieurs questions diplomatiques se trouvent largement instrumentalisées à des fins de politique intérieure : au printemps 1953, les prises de position de Berija en faveur d'une Allemagne «pacifique » qui pourrait ne pas être «socialiste $»^{2}$ déchaînent l'hostilité violente du Présidium ${ }^{3}$ et préfigurent la proche élimination du « traître en puissance ». Quelques mois plus tard, en août, l'accueil plus que critique réservé aux déclarations de Malenkov sur le caractère universel du péril nucléaire atteste déjà de sa marginalisation politique et bientôt de son éviction ${ }^{4}$.

2. Sur cette prise de position iconoclaste, voir James Richter, « Reexamining Soviet policy towards Germany during the Beria interregnum », in The Cold War International History Project, Working Paper, 3, June 1992.

3. Dans ses entretiens avec Čuev, Molotov témoigne de la virulence des échanges qui accueillirent la proposition de Berija, in Félix Tchouev, Conversations avec Molotov, 140 entretiens avec le bras droit de Staline, Paris, Albin Michel, 1995, p. 274-276.

4. Voir sur cette question importante la biographie consacrée par A. G. Malenkov à son père, intitulée O moem otce G. Malenkove, Moscou, NTC, 1992. 
Dans ce contexte particulier, marqué par des querelles de succession, des relations nouvelles se nouent entre le MID et le parti.

Tout en prétendant agir au nom des intérêts du parti et dans le respect des volontés de ce dernier, Molotov s'efforce d'imposer au Présidium ses positions et celles de son administration ; et il connaît de fait un certain succès en amenant le Présidium à entériner des positions élaborées par ses services. Dans le flottement qu'entraînent la disparition de Stalin et la promotion d'une direction collégiale qui n'est pas homogène, les perceptions de Molotov et sa lecture manichéenne des relations internationales pèsent de manière marquée sur les décisions adoptées par le Présidium.

Cette prééminence de Molotov et des services du MID se repère de manière très nette tout au long de l'année 1954. Ainsi, durant la préparation de la conférence quadripartite de Berlin qui se tient en février 1954, les services du MID, dont au premier plan le Troisième département européen en charge des questions allemandes, prônent des positions extrêmement intransigeantes à l'égard de l'Occident, martèlent leur refus de toute amorce de discussion au sujet d'une hypothétique réunification qui se ferait sur la base d'élections prétendues libres et s'affirment, dans les rapports préparatoires qu'ils rédigent à l'intention du Présidium, convaincus par avance de l'échec de la conférence à venir'5. De même, l'organisation de la conférence de Moscou de novembre 19546 est l'occasion pour Molotov de promouvoir, devant les représentants du bloc socialiste et des partis communistes ouest-européens, des thèses staliniennes, dont au premier plan celle d'une Allemagne belliqueuse et agressive contre laquelle il faut à tout prix se prémunir par l'adoption de mesures de sécurité collective et de désarmement.

D’une manière plus générale, durant les années 1953-1954, Molotov et l'administration du MID sont en mesure d'imposer au Présidium un certain nombre de thèmes issus de la guerre froide : une hostilité de principe à l'égard des États-Unis et des États d'Europe occidentale, des critiques virulentes à l'encontre de l'OTAN perçue comme une structure offensive et agressive, un attachement viscéral au glacis territorial est-européen et, plus encore, une inquiétude marquée devant l'évolution de la question allemande : le rapprochement qui, depuis la fin de la guerre, s'opère entre l'Allemagne « occidentale » et l'ensemble des pays occidentaux ne cesse en effet d'alimenter les angoisses des décideurs soviétiques.

Dans le même temps, cette machine diplomatique qui prétend placer sa compétence et son expérience au service du parti reste largement extérieure à lui : dans les années 1953-1955, trois ambassadeurs seulement, les ambassadeurs en poste aux États-Unis, en Grande-Bretagne et en Chine populaire, sont membres du Comité central et ils ne sont que membres candidats. Seul Molotov est alors membre de

5. Cette position se dessine très clairement dans le rapport du 14 janvier 1954, émanant du Troisième département européen et intitulé «Les plans anglo-saxons sur les élections "libres" en Allemagne », et consigné dans les archives du MID, referentura po Germanii, op. 42, por 34 , papka 287, dossier « Sur la conférence de Berlin ».

6. Sur la conférence intitulée «Conférence européenne pour la sauvegarde de la paix et de la sécurité en Europe » qui se tient à Moscou du 29 novembre au 2 décembre 1954, voir les dossiers préparatoires figurant dans les archives du MID, fonds 46, « conférence de Moscou », op. 1, por. 1, delo 1, « Matériaux ». 
plein droit du Comité central, mais il ne saurait évidemment être considéré comme un diplomate de carrière.

Héritée des années 20 et 30, cette séparation étanche entre fonctions diplomatiques et responsabilités au sein du parti est extrêmement utile à Molotov : elle lui permet en effet de placer «à part » l'administration des affaires étrangères, dans une sorte de «bulle » de compétences au moment même où le poids et l'influence de cette dernière sur les décisions du parti sont à leur zénith. Car dès 1955, l'autorité du MID sur le Présidium commence à s'affaiblir.

À partir de 1955, en effet, l'autorité du MID sur le Présidium se fait moins forte. Les membres du Présidium ne sont plus tout à fait novices en matière de politique extérieure et plusieurs d'entre eux parviennent non seulement à affirmer des positions différentes de celles du MID mais plus encore à les faire adopter.

Deux questions majeures ont ainsi servi de test. Au printemps 1955, alors que Molotov y était farouchement opposé et que l'administration centrale du MID jugeait le projet aventureux, Hruščev et Bulganin qui se font les avocats d'un rétablissement des relations soviéto-yougoslaves l'emportent au sein du Présidium. Et à la fin du mois de mai 1955, Hruščev effectue un spectaculaire voyage en Yougoslavie qui, là encore contre l'avis de Molotov, aboutit à la publication d'une déclaration commune soulignant la diversité des voies d'accès au socialisme. La question autrichienne fut encore plus lourde de sens : opposant longuement Molotov et le MID qui refusaient de concéder à l'Autriche toute souveraineté au nom du maintien du sacro-saint glacis territorial stalinien, à Hruščev et à son secrétariat qui voulaient en finir avec cette « séquelle de la guerre froide $»^{7}$, l'affaire se conclut au sein du Présidium à l'avantage de Hruščev. Et désormais, le MID retourne au rôle d'exécutant des volontés du parti. Par la suite, cette réalité ne fait que s'accentuer : au fil des années 1957-1964, l'administration du MID se trouve soumise aux volontés du Présidium et, de plus en plus, aux volontés de Hruščev lui-même.

\section{5-1964, un MID retourné dans l'ombre du Présidium}

En dépit de la nomination en 1957 d'un diplomate expérimenté à la direction du MID, - Andrej Gromyko a été présent aux conférences de Dumbarton Oaks, de Yalta, de San Francisco et de Potsdam, il est auréolé de son prestige d'ancien ambassadeur à Washington et il est membre de plein droit du Comité central depuis la fin de l'année $1956^{8}$ - , la politique extérieure soviétique reste au fil des années 1957-1964 l'apanage d'un Présidium par ailleurs de moins en moins monolithique. En juin 1957, l'élimination politique du groupe anti-parti a permis à Hruščev d'en finir avec la vieille garde stalinienne et de gagner en autorité. Mais en matière de politique étrangère, des nuances, voire des divergences, ne cessent plus de se dessiner au sein du Présidium : à un Mikojan confiant dans le fonctionnement et l'avenir de la coexis-

7. Selon l'expression de Nikita Khrouchtchev, Mémoires inédits, Paris, Belfond, 1991, p. 106.

8. Quatre ans après avoir été nommé membre suppléant en 1952. 
tence pacifique ${ }^{9}$, s'opposent Suslov et Kozlov qui, eux, se distinguent par leur vision inquiète, voire paranoïaque, des relations internationales. Pour eux, les États-Unis, soutenus dans cette voie par les puissances ouest-européennes, sont engagés dans la préparation d'une guerre nucléaire contre l'URSS et la coexistence pacifique n'est qu'un leurre ${ }^{10}$. Toutefois, s'ils parviennent à faire entendre leur point de vue, les deux hommes ne seront pas en mesure de l'imposer à un Présidium qui, jusqu'en 1964, reste acquis aux vues plutôt optimistes de Hruščev.

De fait, entre 1956 et 1964, la primauté de Hruščev sur le plan diplomatique est un fait avéré qu'illustrent la consultation des archives du MID et du parti et la lecture des écrits des diplomates soviétiques contemporains de la période. Dans ses Mémoires, Ot Kollontaj do Gorbačeva. Vospominanija diplomata, le diplomate Aleksandrov-Agentov souligne ainsi :

Hruščev n'était pas homme à permettre à quiconque de formuler à sa place la politique extérieure. [...] Les idées et les initiatives diplomatiques provenaient bien de Hruščev ; en revanche, c'est au ministre des Affaires étrangères et à son appareil que revenait la tâche de les rendre intelligibles, de les corriger, de les étayer et de leur donner forme ${ }^{11}$.

Et plus loin :

Tous les épisodes les plus délicats de notre politique extérieure d'alors - traité sur l'Autriche, rapprochement avec la Yougoslavie et avec l'Inde, propositions à l'ONU sur l'indépendance des peuples colonisés et sur le désarmement général - et nos difficultés - rupture avec la Chine, échec de la conférence de Paris en 1960 et crise de Cuba en 1962 - étaient le fruit de l'intervention personnelle de Hruščev et de ses initiatives dans notre politique extérieure ${ }^{12}$.

Cette prééminence a été particulièrement nette en plusieurs circonstances : dans la gestion de la crise de Berlin de $1958^{13}$ et dans l'affaire de la conférence de Paris de 1960 où, alors que le secrétariat général du MID préparait activement le sommet ${ }^{14}$

9. Voir par exemple ses déclarations dans la Pravda, 18 août 1961.

10. Plusieurs discours attestent de ce thème récurrent. Voir par exemple les déclarations de Kozlov dans la Pravda, 20 septembre 1961 ou bien encore le discours de Suslov reproduit dans la Pravda, 13 mars 1962.

11. Andrej Aleksandrov-Agentov, Ot Kollontaj do Gorbačeva. Vospominanija diplomata (De Kollontaj à Gorbačev. Mémoires d'un diplomate), Moscou, Meždunarodnye Otnošenija, 1994, p. 70-71.

12. Ibid., p. 72.

13. Voir sur ce point les travaux de Hope Harrison, en particulier son article « New archival evidence on the dynamics of Soviet-East German relations and the Berlin crisis, 1958-1961 », in The Cold War International History Project, Working Paper, 5, May 1993.

14. Les archives du MID aujourd'hui déclassifiées et la publication de plusieurs témoignages concordants attestent de l'attachement des diplomates du MID au succès d'une conférence qu'ils ont préparée avec soin. Voir en particulier le témoignage d'Anatoly Dobrynin dans ses Mémoires, In confidence, Moscow's ambassador to America's six Cold War presidents, 19621986, New York, Times Book, Randon House, 1995, p. 42. 
et que le Troisième département européen assaillait Gromyko de rapports préparatoires, Hruščev prend seul l'initiative de la crise internationale qui conduit au sabordage de la conférence. Et elle s'est parfois affichée sans ménagement à l'égard des diplomates de carrière. Aleksandrov-Agentov rapporte ainsi de manière pittoresque, voire cruelle :

À l'automne 1958, devant l'auteur de ces lignes, Gromyko, accompagné de deux de ses collaborateurs, rendit visite à Hruščev, dans son bureau du Comité central, pour lui faire part de ses réflexions concernant nos futures démarches sur la question d'actualité que constituait alors Berlin-Ouest. Andrej Andreevič mit ses lunettes et commença à lire le rapport qu'il avait préparé. Mais soudain Hruščev l'interrompit en lui déclarant : «Attends et écoute ce que je dis, la sténo le prendra en note. Si cela correspond à ce que tu as préparé, c'est bien ; mais si ce n'est pas le cas, eh bien, tu n'auras qu'à mettre ton texte au panier! » Et il commença à dicter (comme toujours de manière confuse et négligée sur le plan de la forme mais de manière assez claire sur le fond), son idée de faire de BerlinOuest une ville libre et démilitarisée ${ }^{15}$.

La primauté du parti et de son Secrétariat en matière de politique extérieure n'a toutefois pas privé le MID de toute initiative : tout au long de la période, le MID dispose en amont du processus décisionnel d'une réelle marge de manœuvre par les rapports et recommandations qu'il présente pour examen au Présidium ou au Secrétariat, par les grilles d'analyse qu'il livre, par la lecture qu'il donne des événements et par les suggestions qu'il émet. Toutefois, le MID n'est pas le seul à remplir cette fonction de conseil et il subit la concurrence d'autres instances qui interviennent, elles aussi, en amont du processus décisionnel. Dans la période khrouchtchevienne, c'est du Département international du Comité central que provient la concurrence la plus vive.

Fondé le 19 mars 1953, le «Département du Comité central du PCUS pour les relations avec les partis communistes étrangers » est à son origine essentiellement chargé des questions de propagande au sein des pays capitalistes. En avril 1956, la dissolution du Kominform en fait une instance majeure du système soviétique qui précède hiérarchiquement le MID ${ }^{16}$ et qui coiffe surtout les relations entre les différents partis communistes d'Europe occidentale et le PCUS. À partir de mars 1955, le Département international est dirigé par Boris Ponomarev ; spécialiste des questions de propagande, Ponomarev, qui a servi dans l'appareil du Kominform de 1936 à 1943 et a été directeur de l'Institut Marx-Engels en 1943-1944, apparaît comme un gardien rigoriste de l'orthodoxie marxiste-léniniste.

Sous la houlette de Ponomarev, le Département international, qui compte une centaine de personnes dans la période khrouchtchevienne, a la responsabilité de la « famille » communiste : il gère l'aide financière accordée aux différents partis communistes occidentaux, mais il est également chargé du développement des

15. A. Aleksandro-Agentov, op. cit.,p. 71.

16. Voir sur ce point le témoignage de Nicolas Polianski, M.I.D. : douze ans dans les services diplomatiques du Kremlin, Paris, Belfond, 1984, p. 34-35. 
mouvements marxistes dans le Tiers-Monde. Or c'est évidemment un premier point qui pose problème au MID car lorsqu'en conformité avec les directives du parti, le Département international choisit de soutenir tel ou tel mouvement plutôt que tel autre, il effectue des choix qui touchent à la sphère diplomatique et empiètent d'une certaine manière sur les intérêts du ministère des Affaires étrangères. De même si, en théorie, les partis indépendantistes relèvent des attributions du Département international tant qu'ils s'inscrivent dans une logique de conquête du pouvoir, et relèvent en revanche de la compétence du MID dès lors qu'ils accèdent au statut de Partis-États, en pratique, la répartition des tâches n'a jamais été facile. Et en plusieurs circonstances, Gromyko s'est plaint de l'existence de deux instances chargées d'élaborer la politique extérieure ${ }^{17}$.

Dans ce contexte - l'autorité prédominante du Présidium dans le processus décisionnel et une concurrence sinon une rivalité avec le Département international en amont de ce processus - la marge de manœuvre de l'administration diplomatique apparaît donc comme plutôt réduite dans la période 1956-1964 : le MID reste bien, avant tout, l'agent d'exécution des volontés du Présidium, voire l'agent d'exécution des volontés khrouchtcheviennes. Cet agent est-il efficace et perçu comme tel ? C'est ce qu'il convient d'observer maintenant.

\section{La machine diplomatique et ses hommes, un outil efficace ?}

Largement marquée par l'héritage stalinien et molotovien de 1953 à 1956, la machine diplomatique tend à s'en émanciper dans la seconde moitié des années 60 , sous l'influence de la « révolution » khrouchtchevienne.

\section{La formation et le fonctionnement de l'appareil diplomatique de 1953 à 1956 , l'empreinte de Molotov}

Dans le sillage des grandes purges, les nominations de 1939-1941 au sein du Narkomindel avaient contribué à la promotion d'une nouvelle génération de diplomates ${ }^{18}$. Jeunes, d'origine modeste, issus du peuple, il s'agissait majoritairement de provinciaux (à près de $80 \%$ ), russes (à plus de $85 \%$ ) qui, dotés d'une expérience professionnelle de techniciens ou d'ingénieurs, étaient passés par l'École supérieure de la diplomatie créée par Molotov pour asseoir leur formation de diplomates.

Ces caractéristiques se modifient de manière sensible à partir des années 19491950 et plus encore à partir de la déstalinisation des années 1953-1954. Cependant la

17. Les témoignages abondent dans ce sens ; voir par exemple celui d'Arkadi Chevtchenko in Rupture avec Moscou, Paris, Payot, 1985,p. 232 : « Un jour que nous parlions de lui [Ponomarev], et de son département, Gromyko s'emporta, disant qu'il ne comprenait pas pourquoi deux instances différentes s'occupaient de politique étrangère. »

18. Voir Teddy J. Uldricks, « The impact of the Great Purges... », art. cit., p. 187-204 et voir l'article de Sabine Dullin dans le présent numéro. 
primauté des Russes demeure et le profil sociologique des nouveaux diplomates reste le même. En revanche les nouveaux venus sont encore plus jeunes que leurs prédécesseurs et ils entrent dans la carrière sans expérience professionnelle, après avoir été formés au MGIMO (Moskovskij Gosudarstvennyj Institut Meždunarodnyh Otnošenij). Cette tendance deviendra prédominante au début des années 1960 et, désormais, le brillant parcours d'Anatolij Dobrynin, secrétaire adjoint de l'ONU de 1957 à 1960 puis ambassadeur aux États-Unis de 1962 à 1988 alors qu'il est initialement diplômé d'un institut aéronautique, apparaîtra comme tout à fait atypique.

La formation des diplomates au sein du MGIMO constitue en effet le fait majeur des années 50. C'est en octobre 1943 qu'au sein de l'Institut d'État de Moscou, une faculté des relations internationales a vu le jour avant de devenir au lendemain de la Seconde Guerre mondiale un institut à part entière, le MGIMO. Cette création s'est inscrite dans un contexte qui n'était pas anodin - celui de l'effervescence diplomatique qui caractérisait la fin de la Seconde Guerre mondiale - et elle témoignait d'un souci nouveau, celui de former en nombre plus grand - deux cents étudiants environ étaient recrutés chaque année - des spécialistes en relations internationales susceptibles soit de travailler directement au sein du MID, soit de rejoindre les autres sphères touchant aux relations avec l'étranger, comme l'Union des associations pour l'amitié entre les peuples, TASS, les maisons d'éditions et les revues destinées à la propagande extérieure.

Dès ses premières années, le recrutement du MGIMO $^{19}$ diffère sensiblement du recrutement à l'École supérieure de la diplomatie. En effet, cette dernière accueillait pour l'essentiel des cadres du parti, des komsomols et des cadres des organisations sociales, envoyés à l'École sur recommandation des instances régionales du parti, c'est-à-dire avant tout sélectionnés sur des critères idéologiques. Le MGIMO ne rompt pas complètement avec cette pratique et il continue d'accueillir des étudiants répondant à ces critères auxquels s'ajoute une minorité d'anciens combattants ${ }^{20}$, mais la tendance nouvelle est au recrutement, au travers d'un concours extrêmement sélectif où la maîtrise d'une langue étrangère s'avère un critère décisif, de candidats de plus en plus jeunes, tout juste sortis de l'enseignement secondaire. Si les étudiants doivent toujours faire preuve d'une parfaite allégeance au système communiste sur ce point, le témoignage sans fard de Georgij Arbatov est particulièrement éloquent ${ }^{21}-$ et s'ils resteront, tout au long des années 50 , sous la haute surveillance

19. Voir le précieux ouvrage Polveka spustja (1948-1998): govorjat pervye vypuskniki MGIMO (Un demi-siècle plus tard (1948-1998) : les premiers élèves sortant du MGIMO témoignent), Moscou, Moskovskij učebnik i kartolitografija, 1998.

20. Environ 60 sur la promotion de 1943 qui comptait 200 étudiants

21. Voir Georgi Arbatov in The system, an insider's life in Soviet Union, New York - Londres, Times Book, Random House, 1992 : «Peu après la grande victoire, une nouvelle vague de persécutions et des campagnes de répression débutèrent. Cette atmosphère était palpable au sein de notre institut. Nous, les étudiants, étions préparés pour des carrières politiques diplomatiques et des postes à l'étranger. En conséquence, nous subissions un examen minutieux et féroce. Nous avions à remplir une très longue et méticuleuse anketa. C'était un document d'une dizaine de pages, comportant toutes sortes de questions déplaisantes sur nous et les membres de notre famille. "Vous ou quelqu'un de votre famille a-t-il appartenu à un 'autre parti' ? (c'est-à-dire 
des autorités politiques ${ }^{22}$, la conformité idéologique ne constitue plus le critère primordial conditionnant le recrutement des futurs diplomates.

Cela étant, cet effort accompli en faveur d'un renouvellement des élites via des procédures de recrutement qui privilégient les compétences des étudiants et non plus leur conformité idéologique ne se trouve pas, dans la période 1953-1956, prolongé par une ouverture quant à l'enseignement délivré.

Les disciplines reines des anciens programmes de l'École supérieure de la diplomatie maintiennent leur position privilégiée dans la nouvelle structure. C'est le cas du droit, dont l'enseignement est confié à des théoriciens autant qu'à des praticiens brillants : l'un d'eux, le professeur Durdinevskij, a fait partie de la délégation soviétique associée aux négociations internationales sur l'élaboration de la charte de l'ONU ${ }^{23}$. Parallèlement, il faut souligner la qualité de l'enseignement des langues étrangères, particulièrement efficace. Alors que les autres enseignements regroupent en moyenne 20 à 25 étudiants, les cours de langue sont limités à 5 ou 6 étudiants et se caractérisent par des méthodes modernes et innovantes pour la période ${ }^{24}$. Enfin, certains enseignants ont un charisme et une envergure qui ne peuvent que fasciner les étudiants ; c'est le cas de Lozovskij qui dispense un enseignement au MGIMO alors qu'il est vice-commissaire du peuple aux Affaires étrangères.

Toutefois, la formation donnée au MGIMO ne va pas sans présenter de sérieuses limites : la vulgate marxiste-léniniste continue de structurer de manière très pesante les enseignements d'histoire, de géographie, de sciences politiques et d'économie. Le Cours abrégé de l'histoire du PCUS, publié en 1948, reste toujours le cadre obligé de référence ${ }^{25}$ dans une vision manichéenne jdanovienne qui combine des éléments agressifs - il s'agit de faire la démonstration bruyante de la supériorité du système socialiste sur tout autre système - et des éléments défensifs, voire paranoïaques, puisque réapparaît avec force le thème de la citadelle socialiste « encerclée et menacée » par les ambitions des impérialistes. Enfin, et plus encore, la formation alors délivrée aux futurs diplomates est complètement close sur ellemême : les étudiants du MGIMO n'ont aucun contact avec les étrangers présents en URSS et leur connaissance directe du monde extérieur est extrêmement limitée ; Jurij Dubinin souligne qu'il lui faut attendre, alors qu'il est déjà spécialisé dans les

à un parti autre que le parti communiste). Vous ou quelque membre de votre famille vous êtesvous trouvé sur des territoires occupés par l'armée allemande? Avez-vous été ou un membre de votre famille a-t-il été prisonnier de guerre ? Avez-vous de la famille à l'étranger ? et si oui, qui ? où ? que font-ils ? Correspondez-vous avec eux ?" et ainsi de suite... Ce document devait être rempli chaque année à l'Institut et que Dieu vous vienne en aide s'il y avait une différence quelconque entre les réponses données d'une année sur l'autre. Pire encore, pratiquement tous les ans, un groupe d'étudiants était arrêté. » p. 33-34.

22. Voir Polveka spustja, op. cit., p. 10.

23. Jurij Dubinin, Diplomatičeskaja byl’ : zapiski posla vo Francii (Histoire diplomatique : mémoires d'un ambassadeur en France), Moscou, ROSSPEN, 1997, p. 17.

24. Une grande importance est accordée à la pratique de la langue orale, sous toutes ses formes, y compris de manière ludique par la mise en scène de pièces de théâtre. Voir Polveka spustja, op. cit., p. 7.

25. Ju. Dubinin, op. cit., p. 13. 
questions françaises ${ }^{26}$, la dernière année de cours au MGIMO pour être autorisé à consulter, dans un dépôt spécial, le journal L'Humanité, tandis qu'il ne peut toujours pas lire les autres journaux français.

Dans ce cadre, la question de la compétence et de l'efficacité de la machine molotovienne se pose avec acuité. Dans ses Mémoires parus en 1994, le diplomate Aleksandrov-Agentov met en valeur un certain nombre de dysfonctionnements, caractéristiques de la période 1945-1956:

Comme je le comprends clairement aujourd'hui, à cette époque, dans leur style de travail et dans tout leur mode de vie, se reflétaient assez clairement de nombreux traits caractéristiques de la machine stalinienne: un centralisme maximal, absolu, un refus de toute liberté d'esprit et de toute initiative « déplacée » venue d'en bas, un secret allant jusqu'à l'absurde et un isolement complet du petit personnel, privé de toute information politique sérieuse ${ }^{27}$.

Et le diplomate de souligner de manière incisive la vacuité des tâches accomplies par des bureaucrates privés de toute information sérieuse :

Des dizaines de personnes travaillaient dur, du matin jusqu'à tard le soir, à la rédaction de papiers n'ayant en fait aucune signification réelle : ces fonctionnaires rédigeaient les annotations des rapports trimestriels et annuels de nos ambassades et de nos missions, inventant fréquemment de toutes pièces, loin de la réalité et de l'atmosphère réelle du pays en question, la « critique » de ces rapports ${ }^{28}$.

Ces dysfonctionnements, en particulier le manque d'ouverture et d'information sur l'extérieur et les conséquences dommageables qui en découlent, n'échappent pas complètement à l'appareil. Dès 1954, le directeur du MGIMO souligne que des aménagements sont nécessaires et que le pays a besoin de diplomates compétents, plus à l'aise dans les rencontres et les négociations internationales :

Notre politique extérieure s'intensifie. Nous avons de plus en plus de contacts. Et ceci n'est qu'un début. Mais comme vous le savez, des travailleurs maîtrisant bien les langues étrangères, nous n'en avons presque pas. Il n'y a pas longtemps, à Genève, à la conférence sur l'Indochine, il est apparu que personne n'était en mesure de fournir une traduction correcte ${ }^{29}$.

Et deux ans plus tard, durant le $\mathrm{xx}^{\mathrm{e}}$ congrès, le discours de Mikojan, alors ministre du Commerce extérieur, s'inscrit à son tour dans cette même perspective en confessant :

Nous sommes sérieusement à la traîne dans l'étude du capitalisme contemporain ; nous n'étudions pas en profondeur les faits et les chiffres; nous nous limitons

26. Il envisagera même de consacrer une thèse à l'histoire du droit public français avant d'être détourné de ce projet par le directeur du MGIMO.

27. A. Aleksandrov-Agentov, op. cit., p. 43-44.

28. Ibid., p. 44-45.

29. Cité par Ju. Dubinin, op. cit., p. 19. 
souvent, par un souci de propagande, à des faits individuels sur les symptômes d'une crise qui approche ou l'appauvrissement des travailleurs, au lieu de tracer une évaluation détaillée et profonde de ce qu'est la vie dans les pays étrangers ${ }^{30}$.

Entre 1953 et 1956, la diplomatie soviétique, à l'image de son ministère des Affaires étrangères, combine donc des éléments paradoxaux sinon contradictoires : des éléments de modernité, c'est-à-dire un recrutement renouvelé, des diplomates de plus en plus jeunes, mais aussi des éléments renvoyant à la pesanteur stalinienne : à savoir un cadre mental jdanovien et des références permanentes à une représentation manichéenne du monde qui n'incite ni au compromis ni à l'ouverture et favorise l'inertie au sein d'un ministère dont les fonctionnaires restent largement dépourvus de toute information fiable sur le monde extérieur. Mais à partir de 1956, ces schémas traditionnels et relativement inefficaces sont brutalement remis en cause par la révolution mentale que Hruščev s'efforce d'imposer à l'appareil dirigeant.

\section{La révolution khrouchtchevienne : nouvelles perceptions, nouveaux regards sur le monde extérieur}

Dès 1955, mais plus encore à partir du XX congrès du PCUS, la coexistence pacifique entre l'Est et l'Ouest devient la « ligne générale de la politique extérieure soviétique $»^{31}$ et se substitue au principe léniniste de l'inéluctabilité des guerres entre pays capitalistes et pays socialistes qui avait jusque-là dominé la lecture soviétique des relations internationales. Le concept de coexistence pacifique témoigne de la volonté soviétique de déplacer la rivalité soviéto-occidentale de la sphère strictement militaire vers la sphère politico-économique. Mais parce qu'elle ne signifie qu'un déplacement de la rivalité Est-Ouest vers d'autres sphères, la coexistence pacifique ne tarde pas à déboucher sur des slogans de plus en plus volontaristes - rattraper les États-Unis - , des ambitions accrues dans le TiersMonde et une course technologique - Spoutnik en 1957, vol habité dans l'espace en 1961- dont l'objectif majeur est d'impressionner l'adversaire et de le convaincre, à tort, de l'existence d'un «missile gap » en faveur de l'URSS. Ce dernier point est fondamental car c'est bien dans la période khrouchtchevienne que, pour la première fois, l'URSS «met en scène » le facteur technologique pour donner l'illusion de sa supériorité et de sa puissance ${ }^{32}$.

30. Cité par Iven B. Neumann, in Russia and the idea of Europe, Londres - New York, Routledge, 1996, p. 133.

31. Voir Jacques Levesque, L'URSS et sa politique internationale de Lénine à Gorbatchev, 2e éd., Paris, A. Colin, 1987, p. 170-171.

32. De fait, si l'URSS khrouchtchevienne a bien entrepris de rattraper son retard, en particulier dans le domaine des missiles à longue portée, elle n'est pas encore parvenue à le combler en 1964. 
Par ailleurs, alors qu'à la fin de la période stalinienne, le facteur idéologique servait essentiellement à conserver des positions acquises - c'est le cas en Europe centrale et orientale - et à tenter d'affaiblir les puissances occidentales grâce aux attaques portées par les PC d'Europe, la période khrouchtchevienne valorise de manière plus importante qu'auparavant l'atout majeur que représente le mouvement communiste international dans le Tiers-Monde. Certes, les décideurs soviétiques ne sont pas dupes des ambiguïtés recelées par les mouvements de libération nationale. Mais l'aventure, à leurs yeux, doit être tentée. Désormais, l'arme idéologique devient un instrument direct au service de la puissance soviétique et plusieurs documents émanant du parti apportent à ce phénomène une justification théorique. Il suffit d'évoquer le rapport présenté par Hruščev sur le programme du parti adopté en juin 1961 par le Comité central. Le texte du premier secrétaire est clair : l'URSS se doit désormais, «au nom de la paix mondiale », d'orchestrer sur tous les continents la lutte contre l'impérialisme en s'appuyant sur toutes les forces progressistes quelles qu'elles soient ${ }^{33}$. La conséquence de cet intérêt accru pour le Tiers-Monde et l'enjeu géopolitique qu'il représente, c'est évidemment l'évolution de la puissance soviétique dont l'influence va désormais au-delà de sa sphère traditionnelle, c'est-à-dire au-delà de la sphère euro-asiatique et s'étend à l'Amérique latine et à l'Afrique, avec la Guinée et le Ghana... Et très tôt, dès 1956, dans le rapport qu'il présente devant le $\mathrm{XX}^{\mathrm{e}}$ congrès, Suslov se fait l'écho de cette aspiration de l'URSS à la mondialisation de son influence et de son rayonnement en déclarant de manière bien caractéristique : «Il n'y a aucun problème international intéressant les peuples du monde dans lequel, durant ces dernière années, l'URSS n'ait été amenée à se prononcer et à la solution duquel elle n'ait participé. » ${ }^{34}$

Armature théorique nouvelle, la coexistence pacifique n'a pas tardé à susciter de nouvelles pratiques au premier rang desquelles celle des sommets bilatéraux et multilatéraux au plus haut niveau et celle des déplacements de plus en plus nombreux des dirigeants soviétiques : il suffit d'évoquer le voyage de Bulganin et de Hruščev à Londres en 1956, le séjour de Mikojan aux États-Unis en janvier 1959, puis celui de Hruščev en septembre 1959 ou bien encore le voyage de Hruščev en France en mai 1960. Mais elle a aussi conduit à des aménagements d'importance dans l'organisation du MID et dans la formation des diplomates : parce qu'elle valorise les contacts avec le monde occidental, la coexistence pacifique impose d'avoir une école diplomatique plus au fait des réalités du monde extérieur et, par là même, plus efficace.

33. Archives du parti communiste de l'Union soviétique, fonds 586, « Documents préparatoires pour le troisième programme du PCUS, 1958-1961 », consultés sur microfilm, Hoover Institution Archives, Stanford University.

34. Rapport de Mihail Suslov au Xx e congrès du PCUS, cité par James Richter, Khrushchev's double bind : international pressures and domestic coalition politics, Baltimore - Londres, Johns Hopkins University Press, 1994, p. 84. 


\section{Formation des diplomates et fonctionnement du MID, premières prises de conscience et premiers aménagements, 1956-1964}

Les exigences nouvelles clairement formulées lors du $\mathrm{xx}^{\mathrm{e}}$ congrès ont été rapidement à l'origine d'aménagements tant sur le plan de la formation des diplomates que sur le plan du fonctionnement du MID.

La première évolution a concerné les enseignements du MGIMO. Progressivement remaniée à partir de 1956-1960, la scolarité au MGIMO est allongée à six ans ; l'enseignement y est dispensé à raison de six à huit heures quotidiennes de cours et de séminaires six jours sur sept ; il gagne en qualité avec un «training » plus intense dans l'apprentissage des langues étrangères et une information plus solide et plus fiable sur les pays étrangers ${ }^{35}$. Cette dernière tient désormais compte de l'apport intellectuel des nouveaux centres de réflexion et d'analyse crés dans le sillage du $\mathrm{Xx}^{\mathrm{e}}$ congrès et dont l'Institut de l'économie mondiale et des relations internationales, l'IMEMO (Institut Mirovoj Ekonomiki i Meždunarodnyh Otnošenij), fondé en avril 1956 et dirigé tout au long de la période khrouchtchevienne par l'économiste Arzumanjan, constitue le fleuron.

C'est en effet au sein de l'IMEMO que sera élaborée une grande partie de la réflexion khrouchtchevienne sur les questions internationales auxquelles l'Union soviétique est confrontée. Si une grande partie de la réflexion élaborée par l'IMEMO sera de plus en plus systématiquement consacrée au Tiers-Monde et aux perspectives de développement socialiste dans les pays nouvellement décolonisés, les questions occidentales ne sont pas laissées de côté, loin s'en faut. Paru en juillet 1957, le premier numéro de la revue MEiMO, Mirovaja Ekonomika i Meždunarodnye Otnošenija, présente ainsi les « dix-sept thèses sur le Marché commun et l'Euratom », document qui, pendant près de cinq ans, va fixer la position officielle de l'URSS sur le problème de l'intégration ouest-européenne.

Ces changements intervenus dans la formation des futurs diplomates se doublent de changements plus timides en matière d'organisation qui visent, là encore, à accroître l'efficacité de la machine diplomatique .

Désormais, le ministre est conseillé par plusieurs vice-ministres des Affaires étrangères et un collège qui réunit les vice-ministres ainsi que les chefs des divisions géographiques et fonctionnelles les plus importantes. Spécialiste des questions européennes, en particulier françaises, Vasilij Kuznecov occupera jusqu'en 1977 le poste de premier vice-ministre. Parmi les six divisions géographiques qui couvrent l'ensemble des pays européens, trois se détachent par leur importance : le Premier département européen coiffe les pays du Benelux, la France et l'Italie. Le Second département est chargé du Royaume-Uni et de l'Irlande et le Troisième, des questions autrichiennes, allemandes et scandinaves. Au fil des années 1956-1964, le Troisième département se montre de loin le plus actif et le plus dynamique avec

35. Le MGIMO devient alors une des écoles les plus prestigieuses du pays. Voir sur ce dernier point le témoignage du diplomate Aleksandr Kaznacheev, Inside a Soviet embassy : experiences of a Russian diplomat in Burma, Philadelphia - New York, J. B. Lipincott Co, 1962, p. 19. 
des diplomates jeunes et brillants, comme Vladimir Semenov et Georgij Puškin. Membres du collège du MID, ils accéderont tous deux au rang de vice-ministres, ce qui témoigne de l'importance alors dévolue par le pouvoir soviétique à la question allemande...

Mieux informés, plus ouverts sur le monde extérieur à partir de 1956-1960, les diplomates soviétiques ont-ils été plus efficaces et en mesure de regagner progressivement la marge de manœuvre et la part d'influence qui avait été la leur dans les années 20 et dans la première moitié des années 30 ? Pas véritablement dans la période khrouchtchevienne car, nouvellement formés, ils n'ont pas encore, à de rares exceptions près, accès aux postes de responsabilité. Mais en revanche, dans la seconde moitié des années 60 et au fil des années 70, c'est bien cette génération de meždunarodniki qui commencera précisément à s'imposer, en particulier au détriment du Département international du Comité central, et qui poussera le parti dans la voie de la détente. La plupart des grands diplomates des années 70, Kovalev, Dubinin, Abrasimov, seront ainsi les «produits » compétents et efficaces des réformes khrouchtcheviennnes.

Favorables à l'ouverture de l'URSS à l'Occident au point qu'on leur donnera l'appellation de zapadniki, c'est-à-dire d'Occidentalistes dans un clin d'œil au $\mathrm{XIX}^{\mathrm{e}}$ siècle qui n'est pas sans signification, ces «nouveaux » diplomates incarneront, à partir de la seconde moitié des années 60, la montée en puissance d'une machine diplomatique dont le crédit, attesté par la confiance dont bénéficie Andrej Gromyko auprès de Brežnev et son statut de membre de plein droit du Politbjuro acquis en 1973, atteindra son apogée dans la seconde moitié des années 70 et la première moitié des années 80 . Avant que ce crédit ne soit à son tour contesté et brutalement remis en cause à l'heure de la perestroïka.

Université de Paris I-Panthéon-Sorbonne

Centre de recherches sur l'histoire des Slaves

mariepierre.rey@wanadoo.fr 